\title{
DEPENDENCE OF THE EMISSION PROPERTIES OF THE GERMANIUM LONE PAIR CENTER ON GE DOPING OF SILICA
}

\author{
A. Alessi ${ }^{1,2}$, S. Agnello ${ }^{2}$, Y. Ouerdane ${ }^{1}$, F.M. Gelardi ${ }^{2}$ \\ ${ }^{1}$ Laboratoire H. Curien, UMR CNRS 5516, Université Jean Monnet, 18 rue du Pr.Benoît Lauras \\ 42000, Saint-Etienne, France. \\ ${ }^{2}$ Dipartimento di Scienze Fisiche ed Astronomiche, Università di Palermo, I-90123 Palermo, \\ Italy. \\ E-Mail:A. Alessi, antonino.alessi@univ-st-etienne.fr
}

\begin{abstract}
We present an experimental investigation regarding the changes induced by the $\mathrm{Ge}$ doping level on the emission profile of the Germanium Lone Pair Center (GLPC) in Ge doped silica. The investigated samples have been produced by the sol-gel method and by plasma activated chemical vapour deposition and have doping level up to $20 \%$ by weight. The recorded photoluminescence spectra evidence that the GLPC emission profile is the same when the Ge content is lower than $\sim 1 \%$ by weight, whereas it changes for higher doping levels. We have also performed Raman scattering measurements that evidence the decrease of the D1 Raman band at $490 \mathrm{~cm}^{-1}$ when the Ge content is higher than $1 \%$ by weight. The data suggest that both changes can be related to matrix modifications. These findings improve the knowledge regarding the matrix effects on the physical proprieties of the point defects and, in particular, for the GLPC they show that variations in their emission properties are induced by the presence of a second $\mathrm{Ge}$ atom close to the defect within a sphere with a radius of about $2 \mathrm{~nm}$.
\end{abstract}

PACS : 74.62.En, 74.25.nd, 78.55.Qr, 76.30.Mi.

\section{INTRODUCTION}

Many studies have been dedicated to the amorphous $\mathrm{SiO}_{2}$ (silica) and to the Ge doped silica materials [1,2]. Silica in fact can be considered as a model system to investigate the amorphous solid state [1-3] and, moreover, it is used for the production of technological devices [1,2]. In particular, the Ge doped silica, because of its enhanced photosensitivity [4], is suitable to obtain non linear effects, as harmonic generation [5,6], or to induce spatial modulation of the refractive index, Fiber Bragg grating [1,7] or anomalous anisotropic light scattering [8], useful to make sensors or other optical devices. The radiation sensitivity of Ge doped silica has been related to the presence of oxygen deficient point defects [1,9] as the twofold coordinated Ge atom [10], also named Germanium Lone Pair Center (GPLC) [11]. The GLPC has also attracted interest because it can be used as a model defect to investigate the embedding matrix properties $[12,13,14,15]$. This defect is suggested to be formed by a Ge atom linked with two oxygen ones, through two single bonds, and having two electrons forming a lone pair [10]. The GLPC is associated with an optical absorption (OA) band and two photoluminescence (PL) bands. The absorption band is peaked at $\sim 5.15 \mathrm{eV}$ (named $\mathrm{B}_{2 \beta}$ band) with a full width at half maximum (FWHM) of $\sim 0.46 \mathrm{eV}$, whereas the two emission bands peaked at $\sim 4.3 \mathrm{eV}$ (called $\alpha_{\mathrm{E}}$ band) and at $\sim 3.2 \mathrm{eV}$ (named $\beta$ band) with FWHM of $\sim 0.43$ and $\sim 0.48 \mathrm{eV}$, respectively $[10,14]$. The $B_{2 \beta}$ band is attributed to the $S_{0} \rightarrow S_{1}$ transition, $S_{0}$ being the ground singlet state and $S_{1}$ the first singlet excited state $[10,14]$. The $\alpha_{\mathrm{E}}$ and the $\beta$ bands are attributed to the $S_{1} \rightarrow S_{0}$ and to the $T_{1} \rightarrow S_{0}$ transitions, respectively, with $\mathrm{T}_{1}$, the first triplet excited state, supplied by an intersystem crossing (ISC) process connecting $S_{1}$ to $T_{1}[10,14]$.

Even if several studies have been dedicated to this activity and different Ge doping levels have been investigated [1,10,16,17], a clear dependence of the GLPC overall PL features on the Ge content has not yet been evidenced. This aspect is fundamental both for the exact comprehension of the defectmatrix interaction and for applications since photosensitivity, for example, has been connected to ISC [1].

To obtain more information on the matrix influence on defect, we have studied Ge doped silica samples with doping levels from $0.1 \%$ to $20 \%$ by weight produced by sol-gel and by Plasma-activated 
Chemical Vapor Deposition (PCVD) techniques. The relation between defects spectral properties and matrix structural features has been investigated through combined OA, PL and Raman measurements.

\section{EXPERIMENTAL}

The samples used in the measurements were produced by both the sol-gel procedure and the PCVD technique. The first have Ge doping levels of $\sim 0.1 \%$ by weight (corresponding to $\sim 1.8 \times 10^{19}$ atoms $/ \mathrm{cm}^{3}$ ), $0.8 \%$ by weight and $12 \%$ by weight, whereas the latter have Ge doping of $\sim 4.5 \%$ and of $\sim 20 \%$ by weight.

The sol-gel samples were produced starting from mixtures of TEOG (germanium tetraethoxide) and TEOS (silicon tetraethoxide) with relative amounts dependent on the required final Ge doping level. The obtained gels were then differently thermally treated to obtain the glasses. The PCVD samples are of commercial origin.

We performed OA measurements in the range from 3 to $6 \mathrm{eV}$ using a double beam Jasco V560 spectrophotometer. PL measurements in the range 2.6 to $5 \mathrm{eV}$ have been recorded using a Jasco FP 6500 spectrofluorometer using a 150 Watt Xenon lamp as source. All the PL spectra have been detected in $45^{\circ}$ back scattering configuration at room temperature and have been corrected for the spectral dispersion and for the detection system efficiency. Raman scattering measurements in the range 300$1200 \mathrm{~cm}^{-1}$ were acquired with a Bruker SENTERRA micro-Raman spectrometer, with a laser excitation light at $532 \mathrm{~nm}$ and a power level of $20 \mathrm{~mW}$. The spectra were recorded in both non confocal and confocal (in different parts of the sample) operation modes using a lower spectral resolution of $5 \mathrm{~cm}^{-1}$. PL spectra and the time decay measurements of the $4.27 \mathrm{eV}$ emission amplitude as a function of temperature were performed using the synchrotron radiation excitation at the SUPERLUMI station on the I-beamline of HASYLAB at DESY (Hamburg). These data were acquired using excitation radiation at about $5.12 \mathrm{eV}$ with a pulse width of $130 \mathrm{ps}$. The emission was selected by a $0.5 \mathrm{~m}$ monochromator (Czerny-Turner mounting) and it was detected by a LN CCD 1100 Princeton Instruments camera for the PL spectra. For the PL time decay measurements the signal was recorded by a photomultiplier (Hamamatsu R2059 model) using 1024 memory channels to scan the 192 ns time interval between two adjacent pulses. For the time decay measurements a dead time of the detection system of about 2 ns was evaluated and only the data recorded for time longer than $2 \mathrm{~ns}$ were considered for the analysis. To perform the measurements at different temperatures from $10 \mathrm{~K}$ to $300 \mathrm{~K}$, a sample chamber equipped with a continuous flow liquid Helium cryostat was used.

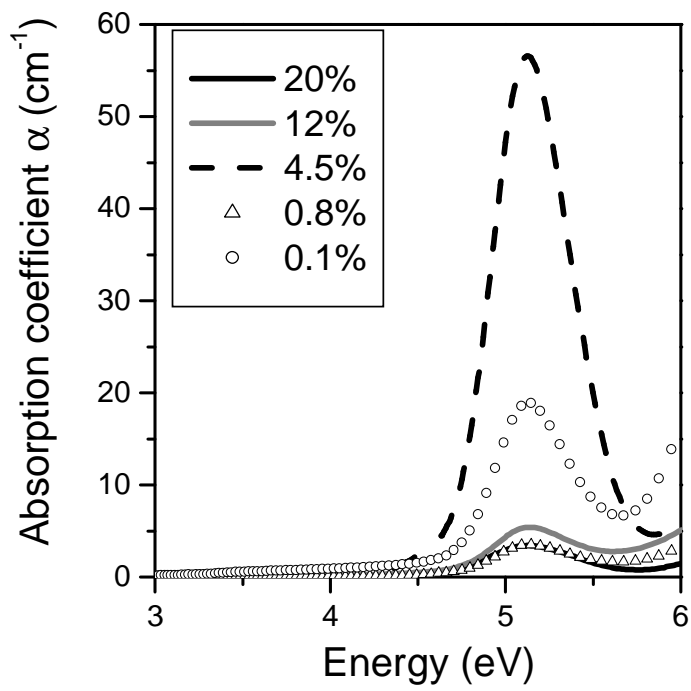

Figure 1: Optical absorption spectra of samples with different Ge doping levels. Sample doped with $20 \%$ of Ge (一), with 12 $\%(-)$, with $4.5 \%(--)$, with $0.8 \%(\Delta)$ and with $0.1 \%(\circ)$, all doping levels are by weight.

\section{RESULTS AND DISCUSSION}

In figure 1, the optical absorption spectra recorded for the samples with doping levels from 0.1 to $20 \%$, are reported. An OA band at about $5.15 \mathrm{eV}$ with the spectral features of the $\mathrm{B}_{2 \beta}$ is always found. The 
amplitude of the absorption, related to the concentration of the GLPC, depends on the sample and on the production procedure as already evidenced [18].

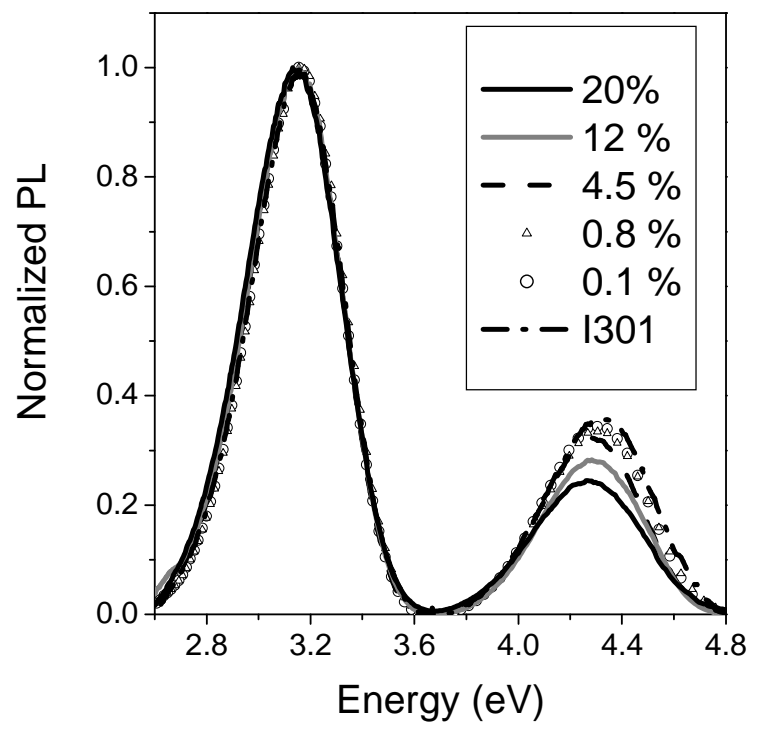

Figure 2: Normalized PL spectra recorded in samples with different Ge doping levels by weight: $20 \%$ of Ge (一), $12 \%(-)$, $4.5 \%(--), 0.8 \%(\Delta), 0.1 \%(\circ)$ and PL spectrum of the natural silica sample I301(一• -). Excitation energy has been fixed at $5.0 \mathrm{eV}$.

In figure 2, the PL spectra of the samples with different doping levels are reported together with the spectrum of a natural silica sample, an Infrasil 301 (I301), containing $~ 0.8 \times 10^{16} \mathrm{GLPC} / \mathrm{cm}^{3}[14,19]$. The spectra are normalized to the emission amplitude at $\sim 3.2 \mathrm{eV}$ and are acquired using the same experimental conditions and excitation energy of $5.0 \mathrm{eV}$.

All the spectra show the two emission bands at $\sim 4.3 \mathrm{eV}$ and at $\sim 3.2 \mathrm{eV}$ characteristic of the GLPC [10, 14]. Up to a doping level of $0.8 \%$ the relative amplitude doesn't depend on the Ge content, whereas above $0.8 \%$ it changes from $\sim 0.36$ to $\sim 0.24$. Furthermore, the position of the $\alpha_{\mathrm{E}}$ band maximum is detected at $\sim 4.27 \mathrm{eV}$ for the $20 \%$ doped sample whereas it is at $\sim 4.32 \mathrm{eV}$ in the less Ge doped samples and in the I301. These findings suggest a dependence of the PL profile on the Ge content. It is worth noting that the absorption coefficient $\alpha$ is lower than $2 \mathrm{~cm}^{-1}$ in the energy range in which the emission bands are detected: as a consequence re-absorption effects cannot affect the emission profile of all the samples. On the other hand, inhomogeneity effects could originate these profile changes acting differently for each sample.
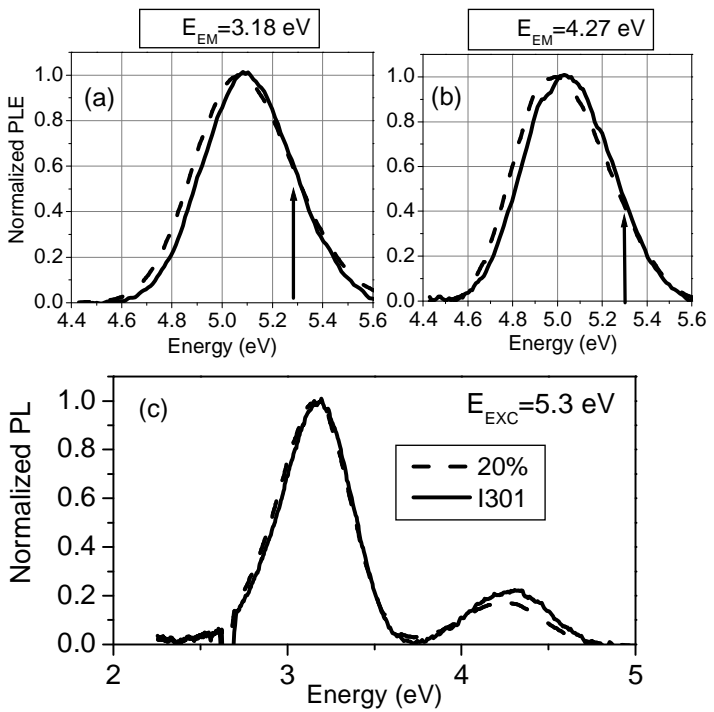

Figure 3: PLE spectra of the sample doped with $20 \%$ by weight of Ge (- - ) and of natural silica sample I301 (-) fixing the emission energy at $3.18 \mathrm{eV}$ (panel a) and at 4.27 (panel b); c) PL spectra of the sample doped with $20 \%$ of Ge (- - ) and of the $\mathrm{I} 301$ (一) fixing the excitation energy at $5.3 \mathrm{eV}$, as evidenced by the arrows in the panels a) and b). 
The PL excitation (PLE) spectra recorded fixing the emission energy at $3.18 \mathrm{eV}$ (figure 3a) and at 4.27 $\mathrm{eV}$ (figure $3 \mathrm{~b}$ ) illustrate minor differences between the I301 and the highest doped sample (20\% by weight of $\mathrm{Ge}$ ). These differences are too low to justify the variation of the PL profiles reported in figure 2. Moreover, as shown in figure $3 \mathrm{c}$, using excitation at $5.3 \mathrm{eV}$, where the PLE profiles are essentially the same (see arrows in figure $3 \mathrm{a}$ and $3 \mathrm{~b}$ ), the differences in the PL spectra are maintained.

To investigate the PL features more quantitatively, we have considered the zero moment $\left(\mathrm{M}_{0}\right)$ of the $\alpha_{\mathrm{E}}$ and $\beta$ bands: $M_{0}=\int_{-\infty}^{\infty} s(E) d E$, where $s$ is the spectral distribution of the band and $E$ indicates the energy. In particular, from the ratio between the $\beta$ band zero moment $\left(M_{0}^{\beta}\right)$ and the $\alpha_{\mathrm{E}}$ band zero moment $\left(M_{0}^{\alpha_{E}}\right)$ it is possible to investigate the Ge doping level effect on the intersystem crossing process once the rate $\left(K_{R}^{F}\right.$ ) of the $\mathrm{S}_{1} \rightarrow \mathrm{S}_{0}$ transition is known. In fact $M_{0}^{\beta} / M_{0}^{\alpha_{E}}$ is equal to $K_{I S C} / K_{R}^{F}, K_{I S C}$ being the ISC relaxation rate from $S_{1}$ to $T_{1}$. This approximation is valid if we can neglect non-radiative channels from $S_{1}$ and $T_{1}$ to $S_{0}$ [14]. This condition has been verified for natural [14] and sol-gel samples with doping level of $0.1 \%$ [20]. For the sol-gel sample $\sim 1 \%$ by weight and for the PCVD sample $20 \%$ by weight we have verified that the sum of $M_{0}^{\beta}$ and $M_{0}^{\alpha_{E}}$ is also independent of T (data not reported).

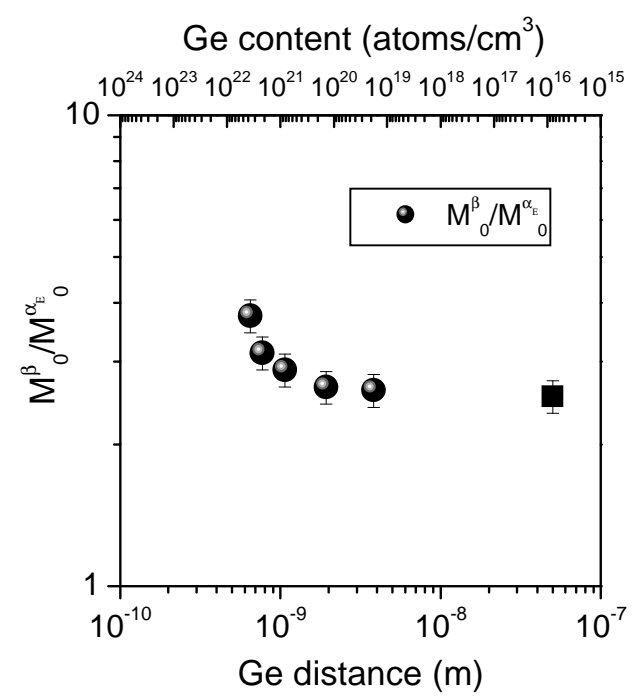

Figure 4: $M_{0}^{\beta} / M_{0}^{\alpha_{E}}$ as a function of the medium Ge distance in Ge-doped samples (circles); the ratio evaluated for the natural silica sample I 301 is represented by the square point.

In figure 4 , we report the ratio $M_{0}^{\beta} / M_{0}^{\alpha_{E}}$, as a function of the medium Ge distance determined from the sample Ge content, assuming that the Ge atoms are homogeneously distributed. The data evidence that no effects are observed for a distance between Ge atoms larger than $2 \times 10^{-9} \mathrm{~m}$ (doping level of 0.8 $\%$ by weight), whereas for lower distances (higher doping) we observe an increase of the ratio.

Lifetime measurements at $4.27 \mathrm{eV}$ show that at $10 \mathrm{~K}$, where $K_{R}^{F}$ is dominant, the decay time is $(8.5 \pm$ $0.5)$ ns for all the studied materials suggesting that $K_{R}^{F}$ is essentially the same for all the doping levels. This unchanged singlet-singlet emission rate, suggests a possible increase of the efficiency of the intersystem crossing process to explain the data of figure 4 .

It is worth noting that the dependency of the PL spectral profile on the Ge content, reported in figure 2, is a feature not dependent on the material preparation technique. In fact, the PL profile of the $12 \%$ by weight Ge-doped sol-gel sample agrees with that of the PCVD samples having doping levels larger than $4 \%$ by weight; whereas the $0.1 \%$ by weight Ge-doped sol-gel sample PL profile is in agreement with the PL profile of the I301 one. Taking into account the data in figure 4 and the above considerations, we can conclude that the GLPC optical proprieties are mainly affected by the local environment, within a sphere of radius of $\sim 2 \mathrm{~nm}$.

Considering this structural effect, it is known that increasing the Ge doping content the Raman spectra of the silica change as a consequence of matrix modifications [21-23]. One of the Raman spectral features that can be used to investigate the Ge content effects on the silica glass matrix is the D1 band, 
peaked at about $490 \mathrm{~cm}^{-1}$. This band is associated to the breathing motion of bridging oxygen atoms in four member rings $[3,22]$. In particular, in previous papers the decrease of the $\mathrm{D}_{1}$ band amplitude on increasing Ge content was associated to the inhibition of ring structure formation [21]. In figure 5a, we report the Raman spectra recorded for the samples with Ge content $4.5 \%, 12 \%$, and $20 \%$ by weight, whereas in the panels b, $\mathrm{c}$ and $\mathrm{d}$ we show magnifications of the spectral region of the D1 band. The D1 decrease in our samples is observed for doping levels above $0.8 \%$, in agreement with previous results [21].
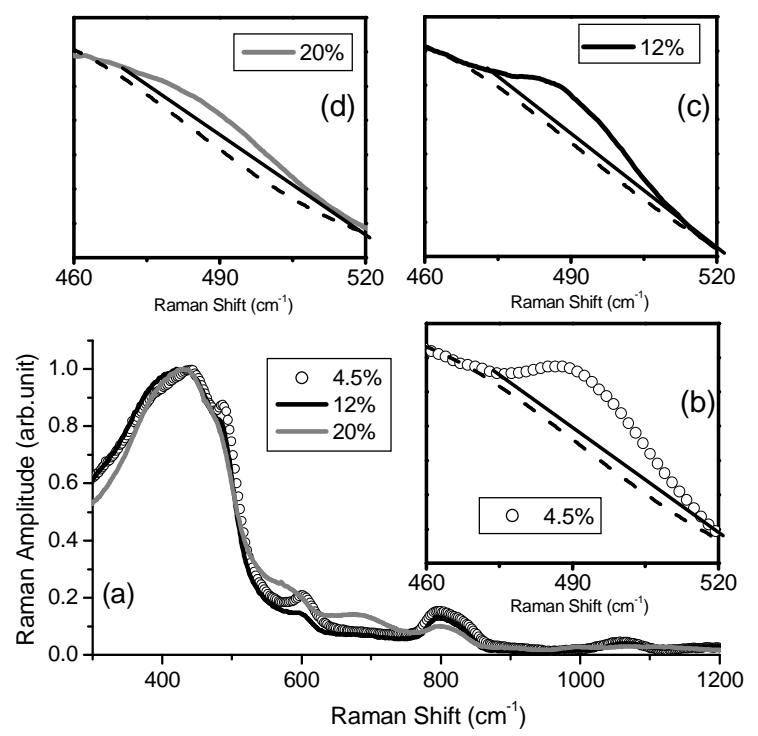

Figure 5: a) Raman spectra of $4.5 \%, 12 \%$ and $20 \%$ by weight Ge doped samples; b), c), d) zoom of the spectral range of the D1 peak recorded in samples doped at $4.5 \% 12 \%$ and $20 \%$ by weight of Ge, respectively. The dashed lines describe the Gaussian bands used to describe the contribution of the Raman band at $440 \mathrm{~cm}^{-1}$ whereas the continuous black lines are tangent to the Raman spectra at $520 \mathrm{~cm}^{-1}$ and cross the spectra at $473 \mathrm{~cm}^{-1}$.
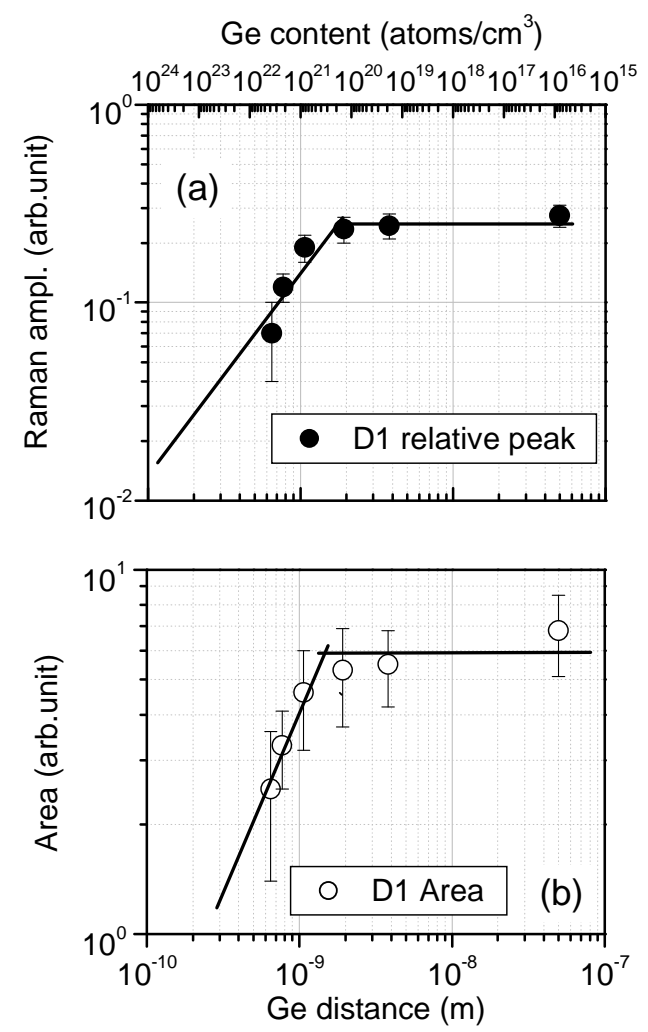

Figure 6: a) D1 relative amplitude as a function of the Ge atoms mean distance; b) area of the D1 band as a function of the Ge atoms mean distance. 
While the D1 band amplitude decreases monotonically with the increasing of the Ge content, a band peaked at $\sim 700 \mathrm{~cm}^{-1}$ appears and becomes clearly distinguishable in the sample with $20 \%$ by weight of Ge. This band is attributed to the Ge-O-Si vibration [22]. As regards, the D2 band peaked at $605 \mathrm{~cm}^{-1}$, attributed to the breathing motion of bridging oxygen atoms in three member rings [3,22], we observe that its amplitude decreases to a doping level of $12 \%$ and then becomes undeterminable because of the large overlap with the bands attributed to Ge-O-Ge vibrations [22]. Finally, we also note that the band peaked at $\sim 800 \mathrm{~cm}^{-1}$, attributed to TO and LO bending modes of Si-O-Si [22], decreases monotonically with the increase of the Ge content. All these features strongly evidence the perturbative role of $\mathrm{Ge}$ atoms on the glass structure.

To make a more quantitative analysis, in figure 6, we report the peak amplitude (panel a) and the area (panel b) of the D1 band as a function of the Ge mean distance. Both spectral feature estimates were obtained averaging the values found after the subtraction of a Gaussian curve that describes the band peaked at $\sim 440 \mathrm{~cm}^{-1}$ (symmetric stretching of the bridging oxygen atoms, $\mathrm{Si}-\mathrm{O}-\mathrm{Si}$, in many member rings [22]) and the values obtained after the subtraction of a straight line that is tangent to the Raman spectrum at $520 \mathrm{~cm}^{-1}$ and that intersects it at $473 \mathrm{~cm}^{-1}$ for each sample (see figure 5). The data in figure 6 evidence that up to a mean distance of $\sim 2 \mathrm{~nm}(0.8 \%$ by weight of $\mathrm{Ge})$ the D1 band is unaffected by the presence of Ge atoms in the glass network. At variance, for lower distance (higher Ge content) an almost linear dependence is observed.

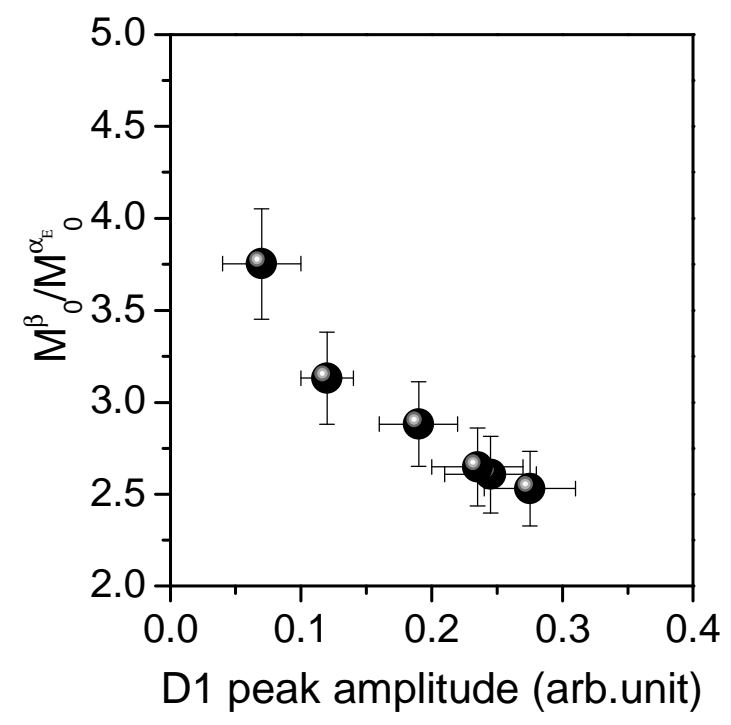

Figure 7: $(\bullet)$ Ratio between the zero moment of the $\beta$ and the $\alpha_{\mathrm{E}}$ PL bands as fuction of the D1 peak.

On the basis of the observation that both $M_{0}^{\beta} / M_{0}^{\alpha_{E}}$ and D1 depend on Ge distance as shown in figure 4 and 6, we can seek a relation between these features. In figure 7, we report $M_{0}^{\beta} / M_{0}^{\alpha_{E}}$ as a function of the $\mathrm{D}_{1}$ amplitude. It can be noted that this ratio decreases with the increase in the D1 peak and that $M_{0}^{\beta} / M_{0}^{\alpha_{E}}$ becomes practically constant for D1 amplitudes higher than 0.23 . The data shown in figure 7 suggest that the changes of population of the rings associated to the $\mathrm{D}_{1}$ band are not directly correlated to the changes of $M_{0}^{\beta} / M_{0}^{\alpha_{E}}$. Being the $3.2 \mathrm{eV}$ band supplied by the phonon assisted ISC process that feeds the $\mathrm{T}_{1}$ state from the $S_{1}$ one [10], it can be concluded that the vibration mode associated to the $D_{1}$ band is not connected to this process. However, it can be guessed that for high Ge concentration, when the Ge distances are below $2 \mathrm{~nm}$, the presence of Ge prevents the formation of the four member rings, as previously suggested [21,23], also introducing modifications in the matrix. On these bases, it can be suggested that the presence of large concentrations of Ge also affects the $S_{1}-T_{1}$ coupling, so modifying the $\mathrm{K}_{\mathrm{ISC}}$ rate.

To investigate the $\mathrm{K}_{\mathrm{ISC}}$ it is important to remember that it has been shown that in the range 140-300 $\mathrm{K}$ it depends on temperature by an Arrhenius law:

$$
K_{I S C}=A \times e^{-\frac{\Delta U}{K_{B} T}}
$$


in which $\Delta \mathrm{U}$ is the activation energy of the process, $\mathrm{K}_{\mathrm{B}}$ is the Boltzmann constant, $\mathrm{T}$ indicates the temperature and $\mathrm{A}$ is a pre-exponential factor $[14,20]$.

In figure 8 , we report the natural logarithm of $M_{0}^{\beta} / M_{0}^{\alpha_{E}}$ as a function of 1000/T normalized to its maximum recorded value: $\left(M_{0}^{\beta} / M_{0}^{\alpha_{E}}\right) /\left(M_{0}^{\beta} / M_{0}^{\alpha_{E}}\right)^{\mathrm{MAX}}=\mathrm{K}_{\mathrm{ISC}} / \mathrm{K}_{\text {ISC }}^{\mathrm{Max}}$ for the $20 \%$ by weight Ge-doped sample. From these data we can estimate $\Delta \mathrm{U}$ by the slope of the linear fitting for $\mathrm{T}>140 \mathrm{~K}$. The result of this procedure indicates that the GLPC present in the sample doped at $20 \%$ by weight have an activation energy of $(85 \pm 10) \mathrm{meV}$. This value is compatible with the value of $(90 \pm 15) \mathrm{meV}$ found in a sample containing $\sim 0.1 \%$ by weight of $\mathrm{Ge}[20]$ and with the value of $\sim 80 \mathrm{meV}$ reported in [14] for the I301. In order to explain the observed increase of the ISC efficiency evidenced in figures 2 and 4, and considering that within experimental uncertainty $\Delta \mathrm{U}$ is unchanged, we can suggest that the $\mathrm{A}$ factor changes increasing with the increase of the Ge doping level. The A factor depends on the matrix disorder through entropic contributions as reported in [20, 24], and its increase does not disagree with the increase of the inhomogeneity of the GLPC environment with doping level. These findings need further experimental investigations, using procedures such as those reported in $[12,15,25]$, to go deeper into inhomogeneity effects. Nevertheless, they suggest that high Ge content affects the properties of point defects and stimulate the extension of the investigation also to other defects such as $\mathrm{Ge}(1), \mathrm{Ge}(2)$ and E'Ge, involved in phenomena like the photosensitivity and the second harmonic generation, in order to better clarify the role of high Ge doping.

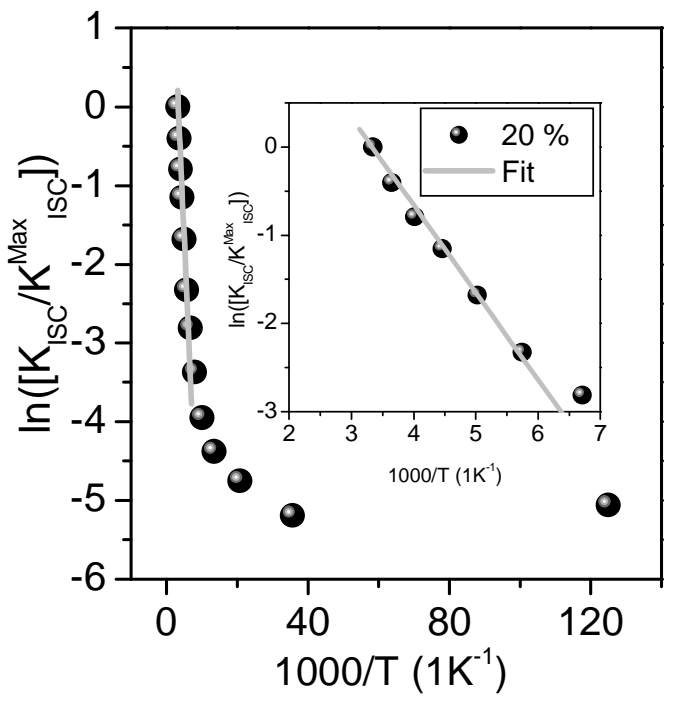

Figure 8: Natural logarithm of the normalized values of $\mathrm{K}_{\mathrm{ISC}}$ as a function of $1000 / \mathrm{T}$ obtained by the $M_{0}^{\beta} / M_{0}^{\alpha_{E}}$ values. In the inset a zoom relative to $\mathrm{T}>140 \mathrm{~K}$ is reported.

\section{CONCLUSIONS}

The reported data show the effects of the matrix changes, evaluated by the Raman spectroscopy, on the optical proprieties of the GLPC and indicate that the GLPC emission proprieties are affected when the medium Ge atom distance is below $\sim 2 \mathrm{~nm}$. These modifications can be attributed to the increase of glass network disorder and to its effect on the ISC process linking the GLPC excited states.

\section{References}

[1] Pacchioni G, Skuja L and Griscom D L (ed) 2000 Defects in $\mathrm{SiO}_{2}$ and related dielectrics: science and technology (Dordrecht: Kluver Academic Publishers).

[2] Devine R A B, Duraud J P and Dooryhée E (ed) 2000 Structure and Imperfections in Amorphous and

Crystalline Silicon Dioxide (New York: Wiley).

[3] Galeener F L and Geissberger A E 1983 Phys. Rev. B 276199.

[4] Hill K O, Fujii Y, Johnson D C and Kawasaki B S 1978 Appl. Phys. Lett. 32647.

[5] Osterberg U and Margulis W 1986 Opt. Lett. 11516. 
[6] Tsai T E, Saifi M A, Friebele E J, Griscom D L and Osterberg U 1989 Opt. Lett. 141023.

[7] Mihailov S J, Smelser C W, Lu P, Walker R B, Grobnic D, Ding H, Henderson G and Unruh J 2003 Opt. Lett. 28995.

[8] Kazansky P G, Inouye H, Mitsuyu T, Miura K, Qiu J, Hirao K and Starrost F 1999 Phys. Rev. Lett. 822199.

[9] Essid M, Albert J, Brebner J L and Awazu K, J. Non-Cryst. Solids 24639.

[10] Skuja L 1992 J. Non-Cryst. Solids 14977.

[11] Awazu K, Kawazoe H and Yamane M 1990 J. Appl. Phys 682713.

[12] D’Amico M, Messina F, Cannas M, Leone M and Boscaino R 2008 J. Phys. Chem. A 112 (47) 12104.

[13] Cannizzo A, Leone M, Gawelda W, Portuondo-Campa E, Callegari A, Van Mourik F and Chergui M 2008 App. Phys. Lett. 93102901.

[14] Nalwa H S (ed) 2001 Silicon-Based Materials and Devices (New York: Academic).

[15] Agnello S, Buscarino G, Cannas M, Messina F, Grandi S, Magistris A 2007 Phys. Stat. Sol. (C) 4934.

[16] Crivelli B, Martini M., Meinardi F, Paleari A and Spinolo G 1996 Solid State Commun. 100651.

[17] Anedda A, Carbonaro C M, Corpino R and Serpi A 1997 J. Non-Cryst. Solids 216, 19.

[18] Alessi A, Agnello S, Grandi S, Parlato A and Gelardi F M 2009 Phys Rev B 80014103.

[19] Agnello S, Boscaino R, Cannas M, Gelardi F M and Leone M 2000 Phys. Rev B 611946.

[20] Agnello S, Boscaino R, Cannas M, Cannizzo A, Gelardi F M, Grandi S and Leone M 2003 Phys. Rev. B 68 165201.

[21] Nian X, Zhisan X and Decheng T 1989 J. Phys. Condens. Matter 16343.

[22] Henderson G S, Neuville D R, Cochain B and Cormier L 2009 J. Non-Cryst. Solids 355468.

[23] Majérus O, Cormier L, Neuville D R, Galoisy L and Calas G 2008 J. Non-Cryst. Solids 3542004.

[24] Cannizzo A, Leone M, Boscaino R, Paleari A, Chiodini N, Grandi S and Mustarelli P 2006 J. Non-Cryst. Solids 3522082.

[25] D’Amico M, Messina F, Cannas M, Leone M and Boscaino R 2008 Phys. Rev. B 78014203. 